\title{
Approximations to the Normal Probability Distribution Function using Operators of Continuous-valued Logic
}

\author{
József Dombi ${ }^{a}$ and Tamás Jónás ${ }^{b}$
}

\begin{abstract}
In this study, novel approximation methods to the standard normal probability distribution function are introduced. The techniques presented are founded on applications of certain operators of continuous-valued logic. It is demonstrated here that application of the averaging Dombi conjunction operator to two symmetric Sigmoid fuzzy membership functions results in a function that is identical with Tocher's approximation to the standard normal probability distribution function. Next, an approximation connected with a unary fuzzy modifier operator is discussed. Namely, the so-called Kappa function is applied for constructing a novel probability distribution function. It is shown here that the asymptotic Kappa function is just the Sigmoid function and the proposed Quasi Logistic probability distribution function can be utilized to approximate the standard normal probability distribution function. It is also explained how the new probability distribution function is connected with the generator function of Dombi operators. The proposed approximation formula is very simple as it has only one constant parameter. It does not include any exponential term, but has a good approximation accuracy and fulfills certain requirements that only a few of the known approximation formulas do.
\end{abstract}

Keywords: continuous logic, Dombi operators, sigmoid function, normal probability distribution, approximation

\section{Introduction}

The normal probability distribution plays a significant role in probability theory and mathematical statistics. Owing to the central limit theorem, it has an extremely wide range of applications in many areas of sciences. The fact that the

\footnotetext{
${ }^{a}$ Department of Computer Algorithms and Artificial Intelligence, University of Szeged, Árpád tér 2, H-6720 Szeged, Hungary, E-mail: dombi@inf.u-szeged.hu

${ }^{b}$ Institute of Business Economics, Eötvös Loránd University, Szép utca 2., H-1053 Budapest, Hungary, E-mail: jonas@gti.elte.hu
} 
cumulative distribution function of the standard normal random variable cannot be expressed in a closed form and the practical needs for computing its values provided the motivations for researchers and practitioners over the last seven decades to approximate the standard normal probability distribution function. These research efforts resulted in an extremely wide range of approximations with many applications.

In this paper, we will introduce approximations to the standard normal probability distribution function that are connected with the well-known Dombi operators in continuous-valued logic. Firstly, we will utilize the averaging Dombi conjunction operator to construct a probability density function from two Sigmoid functions. We will show that this approximation method results in a probability distribution function that is identical with Tocher's approximation from 1963 [29]. Secondly, we will introduce the Kappa function and based on this function, we will construct the Quasi Logistic probability distribution function. We will show that the asymptotic Kappa function is just the Sigmoid function and using this result, we will also show how the Quasi Logistic probability distribution function can be utilized for approximating the standard normal probability distribution function. Here, we will point out how the proposed probability distribution function is connected with the generator function of Dombi operators and with the Kappa function-based uniary operator that can be utilized as a general fuzzy modifier operator. The novelty of our methods lies in the fact that some mathematical constructions of continuousvalued logic can be successfully utilized to construct approximations to probability distribution functions.

Many known approximations to the standard probability distribution function focus mainly on the approximation accuracy, and so these methods result in highly accurate functions, without taking some other aspects of the approximation into account. It should be mentioned here that we require our approximations to meet expectations that are based on certain theoretical and practical considerations. These expectations are simplicity and accuracy, asymptotic equality of the approximator function to the standard normal distribution function to first order at zero, symmetry of probability density function and a direct connection between the density and distribution functions.

Finally, we propose the use of the following probability distribution function, which is a special case of the Quasi Logistic distribution function, to approximate the standard normal probability distribution function:

$$
\Phi_{\kappa, \pi}(x)= \begin{cases}0, & \text { if } x \leq-\pi \\ \frac{1}{1+\left(\frac{\pi-x}{\pi+x}\right)^{\sqrt{2 \pi}},} & \text { if } x \in(-\pi,+\pi) \\ 1, & \text { if } x \geq+\pi .\end{cases}
$$

We call the function $\Phi_{\kappa, \pi}(x)$ the Dombi-Jónás probability distribution function. It has only one constant parameter, which is the number $\pi$, while its maximum absolute approximation error over the set of real numbers is $2.36 \cdot 10^{-3}$. Note that 
there are just a few known approximations with a single constant parameter in this accuracy range (e.g. [26], [20], [1], [13]), and all these approximations include exponential terms, while ours does not contain any and has a very simple form. It should also be added that the probability density function $\phi_{\kappa, \pi}(x)$ can be directly expressed in the terms of the probability distribution function $\Phi_{\kappa, \pi}(x)$ without differentiating it.

In many practical applications, the value of the standard normal probability distribution function for an argument being less than -3 or greater than +3 is considered to be zero and one, respectively, although the probability distribution does not take these values. The proposed $\Phi_{\kappa, \pi}(x)$ approximation has the value of zero, if $x \leq-\pi$, and it has the value of 1 , if $x \geq+\pi$, so the function $\Phi_{\kappa, \pi}(x)$ may be viewed as an alternative, with bounded domain, to the standard normal probability distribution function.

The remaining part of the paper is organized as follows. In Section 2, we will review some notable approximations to the standard normal probability distribution function. Next, in Section 3, we will set our approximation criteria and introduce novel approximation methods that are connected with the Dombi operators. Lastly, in Section 4, we will summarize our approximation results and draw some key conclusions about the proposed Quasi Logistic probability distribution function.

\section{Approximations to the Standard Normal Probability Distribution Function}

Now, we will give a short review of the techniques that are widely used for approximating the standard normal probability distribution function and enumerate some notable approximations that have been constructed in the last seven decades.

We will use the common notations $\phi(x)$ and $\Phi(x)$ for the probability density function and probability distribution function of the standard normal random variable, respectively. That is,

$$
\phi(x)=\frac{1}{\sqrt{2 \pi}} \mathrm{e}^{-\frac{x^{2}}{2}} ; \Phi(x)=\int_{-\infty}^{x} \phi(t) \mathrm{d} t .
$$

The approximation methods available in the literature can be categorized into two main approach categories [21]. One category is the group of approximations that are based on numerical methods, while the other category contains methods that are founded on ad-hoc approximations.

The numerical methods are typically based on numerical integration techniques, various power series, expansions in Hermite or Chebyshev polynomials and continued fraction expansions (e.g. [6], [18], [22], [25], [7]). In general, these methods can yield a high-level approximation accuracy, but require complex computations.

The ad-hoc approximation methods typically utilize an a priori selected parametric function and apply various mathematical techniques to estimate the parameters in order minimize the approximation error. Matic et al. [21], Soranzo 
and Epure [28] and Yerukala and Boiroju [32] gave comprehensive overviews of the approximation formulas in their papers. Here, without striving for completeness, we enumerate some notable approximation formulas and indicate their maximum absolute errors (MAE).

1. Pólya (1949) [26]: $\Phi(x) \approx \frac{1+\sqrt{1-\mathrm{e}^{-2 x^{2} / \pi}}}{2} ; M A E=3.15 \cdot 10^{-3}$

2. Hart (1957) [15]: $\Phi(x) \approx \frac{1}{\sqrt{2 \pi}} \frac{\mathrm{e}^{-2 x^{2} / \pi}}{x+0.8 \mathrm{e}^{-0.4 x}} ; M A E=4.30 \cdot 10^{-3}$

3. Tocher $(1963)[29]: \Phi(x) \approx \frac{\mathrm{e}^{2 \sqrt{2 / \pi}}}{1+\mathrm{e}^{2 \sqrt{2 / \pi}}} ; M A E=1.77 \cdot 10^{-2}$

4. Zelen \& Severo (1964) [34]: $\Phi(x) \approx 1-\left(a_{1} t-a_{2} t^{2}+a_{3} t^{3}\right) \frac{\mathrm{e}^{-\frac{x^{2}}{2}}}{\sqrt{2 \pi}}$, where $t=(1+0.33267 x)^{-1}, a_{1}=0.4361836, a_{2}=0.1201676$, $a_{3}=0.937298 ; M A E=1.15 \cdot 10^{-5}$

5. Hart (1966) $[16]: \Phi(x) \approx 1-\frac{\mathrm{e}^{-\frac{x^{2}}{2}}}{\sqrt{2 \pi} x}\left(1-\frac{\frac{\sqrt{1+b x^{2}}}{1+a x^{2}}}{P_{0}+\sqrt{P_{0} x^{2}+\mathrm{e}^{-\frac{x^{2}}{2}} \frac{\sqrt{1+b x^{2}}}{1+a x^{2}}}}\right)$, where $a=\frac{1+\sqrt{1-2 \pi^{2}+6 \pi}}{2 \pi}, b=2 \pi a^{2}$ and $P_{0}=\sqrt{\pi / 2} ; M A E=5.23 \cdot 10^{-5}$

6. Page (1977) [24]: $\Phi(x) \approx \frac{1}{2} \frac{1}{1+\tanh (y)}$, where $y=\sqrt{\frac{2}{\pi}} x\left(1+0.044715 x^{2}\right)$; $M A E=1.79 \cdot 10^{-4}$

7. Hamaker (1978) [14]: $\Phi(x) \approx 1-\frac{1}{2}\left(1-\sqrt{1-\mathrm{e}^{-y^{2}}}\right)$, where $y=0.806 x(1-0.018 x) ; M A E \approx 6.23 \cdot 10^{-4}$

8. $\operatorname{Lin}(1989)[19]: \Phi(x) \approx 1-\frac{1}{2} \mathrm{e}^{-0.717 x-0.416 x^{2}} ; M A E=6.59 \cdot 10^{-3}$

9. Norton $(1989)[23]: \Phi(x) \approx \begin{cases}1-\frac{1}{2} \mathrm{e}^{-0.717 x-0.416 x^{2}}, & \text { if } 0 \leq x \leq 2.7 \\ \frac{1}{\sqrt{2 \pi} x} \mathrm{e}^{-\frac{x^{2}}{2}}, & \text { if } x>2.7\end{cases}$ $M A E=8.07 \cdot 10^{-3}$

10. $\operatorname{Lin}(1990)[20]: \Phi(x) \approx 1-\frac{1}{1+\mathrm{e}^{4.2 \pi \frac{x}{9-x}}}$, where $0 \leq x<9 ; M A E=6.69 \cdot 10^{-3}$

11. Bagby (1995) [2]: $\Phi(x) \approx \frac{1}{2}+\frac{1}{2} \sqrt{1-\frac{1}{30}\left(7 \mathrm{e}^{-x^{2} / 2}+16 \mathrm{e}^{-x^{2}(2-\sqrt{2})}+\left(7+\frac{\pi}{4} x^{2}\right) \mathrm{e}^{-x^{2}}\right)} ;$ $M A E \approx 3.00 \cdot 10^{-5}$

12. Waissi \& Rossin (1996) [31]: $\Phi(x) \approx \frac{1}{1+\mathrm{e}^{-\sqrt{\pi}\left(0.9 x+0.0418198 x^{3}+0.0004406 x^{5}\right)}} ; M A E=4.37 \cdot 10^{-5}$

13. Bryc (2002) [4]: $\Phi(x) \approx \frac{x^{2}+a_{1} x+a_{2}}{\sqrt{2 \pi} x^{3}+b_{1} x^{2}+b_{2} x+2 a_{2}} \mathrm{e}^{-\frac{x^{2}}{2}}$, where $a_{1}=5.575192695, a_{2}=12.77436324, b_{1}=14.38718147$, $b_{2}=31.53531977 ; M A E=1.87 \cdot 10^{-5}$ 
14. Shore (2005) [27]: $\Phi(x) \approx \frac{1+g(-x)+g(x)}{2}$, where $g(x)=\mathrm{e}^{\left.-\log 2 \mathrm{e}^{\alpha /\left(\lambda / S_{1}\right)\left(\left(1+S_{1} x\right)\left(\lambda / S_{1}\right)\right.}-1\right)+S_{2} x}$ $\lambda=0.61228883, S_{1}=0.11105481, S_{2}=0.44334159, \alpha=6.37309208$; $M A E \approx 10^{-7}$

15. Aludaat and Alodat (2008) [1]: $\Phi(x) \approx \frac{1}{2}+\frac{1}{2} \sqrt{1-\mathrm{e}^{-\sqrt{\pi / 8} x^{2}}}$; $M A E=1.97 \cdot 10^{-3}$

16. Bowling et al. (2009) [3]: $\Phi(x) \approx \frac{1}{1+\mathrm{e}^{-\left(0.07056 x^{3}+1.5976 x\right)}} ; M A E=1.4 \cdot 10^{-4}$

17. Yerukala et al. (2011) [33]:

$\Phi(x) \approx \begin{cases}0.5-1.136 H_{1}+2.47 H_{2}-3.013 H_{3}, & \text { if } 0 \leq x \leq 3.36 \\ 1, & \text { if } x>3.36,\end{cases}$

where $H_{1}=\tanh (-0.2695 x), H_{2}=\tanh (0.5416 x)$ and $H_{3}=\tanh (0.4134 x)$; $M A E=1.25 \cdot 10^{-3}$

18. Vazquez-Leal et al. (2012) [30]: $\Phi(x) \approx \frac{1}{2} \tanh \left(\frac{179 x}{23}-\frac{11}{2} \arctan \left(\frac{37 x}{294}\right)\right)+\frac{1}{2}$; $M A E \approx 1.00 \cdot 10^{-6}$

19. Choudhury (2014) [5]: $\Phi(x) \approx 1-\frac{1}{\sqrt{2 \pi}} \frac{\mathrm{e}^{-\frac{x^{2}}{2}}}{0.226+0.64 x+0.33 \sqrt{x^{2}+3}}$;

$M A E=1.93 \cdot 10^{-4}$

20. Yerukala \& Boiroju (2015) [32]: $\Phi(x) \approx 1-\frac{\mathrm{e}^{-\frac{x^{2}}{2}}}{\frac{44}{79}+\frac{8}{5} x+\frac{5}{6} \sqrt{x^{2}+3}}$; $M A E=1.10 \cdot 10^{-4}$

21. Yerukala \& Boiroju (2015) [32]: $\Phi(x) \approx w \Phi_{1}(x)+(1-w) \Phi_{2}(x)$, where $x>0, w=0.268, \Phi_{1}(x)$ is the approximation by Hart (1966) and $\Phi_{2}(x)$ is the approximation by Bryc $(2002) ; M A E=7.54 \cdot 10^{-6}$

22. Matic et al. (2016) [21]:

$\Phi(x) \approx \frac{1}{2}+\frac{\operatorname{sgn}(x)}{2} \sqrt{1-\mathrm{e}^{-\frac{2 x^{2}}{\pi}\left(1+\gamma_{2} x^{2}+\gamma_{4} x^{4}+\gamma_{6} x^{6}+\gamma_{8} x^{8}+\gamma_{10} x^{10}\right)}}$,

where $\gamma_{2}=-\frac{1}{3}+\frac{1}{\pi} ; \gamma_{4}=\frac{7}{90}-\frac{2}{3 \pi}+\frac{4}{3 \pi^{2}} ; \gamma_{6}=-\frac{1}{70}+\frac{4}{15 \pi}-\frac{4}{3 \pi^{2}}+\frac{2}{\pi^{3}}$;

$\gamma_{8}=\frac{83}{37800}-\frac{76}{945 \pi}+\frac{34}{45 \pi^{2}}-\frac{8}{3 \pi^{3}}+\frac{16}{5 \pi_{8}^{4}}$

$\gamma_{10}=-\frac{73}{249480}+\frac{283}{14175 \pi}-\frac{178}{567 \pi^{2}}+\frac{88}{45 \pi^{3}}-\frac{16}{3 \pi^{4}}+\frac{16}{3 \pi^{5}}$;

$M A E=5.79 \cdot 10^{-6}$

23. Eidous and Al-Salman (2016) [13]: $\Phi(x) \approx \frac{1}{2}\left(1+\sqrt{\mathrm{e}^{-5 / 8 x^{2}}}\right)$;

$M A E=1.81 \cdot 10^{-3}$

Based on the above approximation formulas, we may state that the accuracy of approximations increases with the complexity of formulas and with the number of parameters they possess. 


\section{Novel Methods based on Operators of Continuous-valued Logic}

Fist of all, we will lay down some expectations that we require from approximations and use these criteria to evaluate our results and compare them with some well-known ones. Next, we will introduce the Dombi operators that are familiar in continuous-valued logic and construct novel approximation methods that are connected with these operators.

\subsection{Expectations towards Our Approximations}

The most basic expectation towards an approximation is that it is sufficiently accurate. In the literature, there are many approximations to the standard probability distribution function that focus mainly on the approximation accuracy. These efforts have resulted in highly accurate functions, without taking some other features of the approximation into account. Here we set some criteria - driven by theoretical and practical considerations - that we require our approximations to meet.

Simplicity and accuracy. The approximation functions should have a simple, easily computable formula, and the approximation accuracy should meet the requirements of practical applications.

Identity to first order at zero. Let $F(x)$ be an approximating function to the standard normal probability distribution function. We require $F(x)$ to be a probability distribution function and meet the following criteria:

$$
\begin{gathered}
F(0)=\Phi(0)=0.5 \\
\left.\frac{\mathrm{d} F(x)}{\mathrm{d} x}\right|_{x=0}=\left.\frac{\mathrm{d} \Phi(x)}{\mathrm{d} x}\right|_{x=0}=\left.\phi(x)\right|_{x=0}=\frac{1}{\sqrt{2 \pi}} .
\end{gathered}
$$

Symmetry. Since the probability density function $\phi(x)$ is an even function, $\Phi(-x)=1-\Phi(x)$ holds for any $x \in \mathbb{R}$. We require the approximation $F(x)$ to have the same feature; that is, $F(-x)=1-F(x)$ for any $x \in \mathbb{R}$. Note that if $F(x)$ satisfies the $F(-x)=1-F(x)$ requirement, then the approximation error function $\delta(x)=\Phi(x)-F(x)$ is an odd function, and so the curve of $|\Phi(x)-F(x)|$ is symmetric with respect to the vertical axis.

Direct connection between the density and distribution functions. In practice, it may be useful, if the probability distribution function can be expressed by the probability density function without integration, and vice versa, if the probability density function can be expressed by the probability distribution function without differentiation. Hence, we prefer the approximations that result in probability density and distribution functions with a direct connection between them; that is, one can be expressed by the other one in a closed form. 
It is worth emphasizing that only a few of the known approximations listed in Section 2 meet all the requirements we demanded. In general, the more complex an approximation formula is, the less of our criteria it meets. However, the approximations with more complex formulas and higher number of constant parameters result in a higher approximation precision. Note that many of the known approximations work just with positive values of variable $x$ and let the user compute the approximating function value by using the $\Phi(-x)=1-\Phi(x)$ equation for negative values of $x$.

\subsection{Dombi Operators in Continuous-valued Logic}

Here, we will introduce the Dombi operator class that can be utilized for implementing the conjunction and disjunction operations in continuous-valued logic [8], [10].

Definition 1. The Dombi conjunction and disjunction operator in continuousvalued logic is given by

$$
o_{\alpha}(\mathbf{x})=\frac{1}{1+\left(\sum_{i=1}^{n}\left(\frac{1-x_{i}}{x_{i}}\right)^{\alpha}\right)^{1 / \alpha}} \text { and } \bar{o}_{\alpha}(\mathbf{x})=\frac{1}{1+\left(\frac{1}{n} \sum_{i=1}^{n}\left(\frac{1-x_{i}}{x_{i}}\right)^{\alpha}\right)^{1 / \alpha}},
$$

where $\mathbf{x}=\left(x_{1}, x_{2}, \ldots, x_{n}\right)$, and $x_{1}, x_{2}, \ldots, x_{n}$ are continuous-valued logic variables.

If $\alpha>0$, then the Dombi operator is a conjunction operator; if $\alpha<0$, then it is a disjunction operator. Here, we will use the Dombi conjunction operators with two operands and $\alpha=1$ :

$$
\begin{gathered}
c\left(x_{1}, x_{2}\right)=\left.o\left(x_{1}, x_{2}\right)\right|_{\alpha=1}= \begin{cases}0, & \text { if } x_{1}=0 \text { or } x_{2}=0 \\
\frac{1}{1+\frac{1-x_{1}}{x_{1}}+\frac{1-x_{2}}{x_{2}},}, & \text { otherwise, }\end{cases} \\
\bar{c}\left(x_{1}, x_{2}\right)=\left.\bar{o}\left(x_{1}, x_{2}\right)\right|_{\alpha=1}= \begin{cases}0, & \text { if } x_{1}=0 \text { or } x_{2}=0 \\
\frac{1}{1+\frac{1}{2}\left(\frac{1-x_{1}}{x_{1}}+\frac{1-x_{2}}{x_{2}}\right)}, & \text { otherwise, }\end{cases}
\end{gathered}
$$

where $x_{1}$ and $x_{2}$ are two continuous-valued logic variables. We call $\bar{c}$ the averaging Dombi conjunction operator. Note that operation $c$ is not idempotent, while $\bar{c}$ may be viewed as an idempotent variant of $c$.

Remark 1. Based on the general representation theorem [9],

$$
o(\mathbf{x})=f^{-1}\left(\sum_{i=1}^{n} f\left(x_{i}\right)\right) \text { and } \bar{o}(\mathbf{x})=f^{-1}\left(\frac{1}{n} \sum_{i=1}^{n} f\left(x_{i}\right)\right)
$$


are strict operators, if $f(x)$ is a strictly monotone function, where $\mathbf{x}=$ $\left(x_{1}, x_{2}, \ldots, x_{n}\right)$, and $x_{1}, x_{2}, \ldots, x_{n}$ are continuous-valued logic variables. If we apply the function

$$
f(x)=f_{\alpha}(x)=\left(\frac{1-x}{x}\right)^{\alpha}
$$

to $o(\mathbf{x})$ and $\bar{o}(\mathbf{x})$, then we get the operators $o_{\alpha}(\mathbf{x})$ and $\bar{o}_{\alpha}(\mathbf{x})$, respectively. That is, $f_{\alpha}(x)$ is the generator function of Dombi conjunction and disjunction operators.

In fuzzy logic, the linguistic modifiers like "very", "more or less", "somewhat", "rather" and "quite" over fuzzy sets that have strictly monotonously increasing or decreasing membership functions can be modeled by the following unary operator called the Kappa function [11].

Definition 2. The Kappa modifier operator (Kappa function) is given by

$$
\kappa_{\nu, \nu_{0}}^{(\lambda)}(x)=\frac{1}{1+\frac{1-\nu_{0}}{\nu_{0}}\left(\frac{\nu}{1-\nu} \frac{1-x}{x}\right)^{\lambda}},
$$

where $\nu, \nu_{0} \in(0,1), \lambda \in \mathbb{R}$, and $x$ is a continuous-valued logic variable.

In Section 3.4, we will use a special form of the unary modifier operator in (9) to construct a probability distribution function.

\subsection{The Sigmoid Function and Some of Its Basic Properties}

Since we will use the Sigmoid function to construct probability density and probability distribution functions, here we will introduce it and some of its main properties.

Definition 3. The Sigmoid function $\sigma^{\left(\lambda_{\sigma}\right)}(x)$ with the parameter $\lambda_{\sigma}$ is given by

$$
\sigma^{\left(\lambda_{\sigma}\right)}(x)=\frac{1}{1+\mathrm{e}^{-\lambda_{\sigma} x}},
$$

where $\lambda_{\sigma} \in \mathbb{R}, \lambda_{\sigma} \neq 0, x \in \mathbb{R}$.

Note that the Sigmoid function is also known as the Logistic function. The main properties, such as the range, continuity, monotonicity, limits, role of the parameter and convexity of the Sigmoid function $\sigma^{\left(\lambda_{\sigma}\right)}(x)$ are as follows.

Range. The range of $\sigma^{\left(\lambda_{\sigma}\right)}(x)$ is the interval $(0,1)$.

Continuity. $\sigma^{\left(\lambda_{\sigma}\right)}(x)$ is a continuous function in $\mathbb{R}$.

\section{Monotonicity.}

- If $\lambda_{\sigma}>0$, then $\sigma^{\left(\lambda_{\sigma}\right)}(x)$ is strictly monotonously increasing

- If $\lambda_{\sigma}<0$, then $\sigma^{\left(\lambda_{\sigma}\right)}(x)$ is strictly monotonously decreasing 
Limits. Function $\sigma^{\left(\lambda_{\sigma}\right)}(x)$ takes neither the value zero, nor the value 1 , as these are its limits:

$$
\begin{aligned}
& \lim _{x \rightarrow+\infty} \sigma^{\left(\lambda_{\sigma}\right)}(x)= \begin{cases}1, & \text { if } \lambda_{\sigma}>0 \\
0, & \text { if } \lambda_{\sigma}<0,\end{cases} \\
& \lim _{x \rightarrow-\infty} \sigma^{\left(\lambda_{\sigma}\right)}(x)= \begin{cases}1, & \text { if } \lambda_{\sigma}<0 \\
0, & \text { if } \lambda_{\sigma}>0 .\end{cases}
\end{aligned}
$$

Role of the parameter. The parameter $\lambda_{\sigma}$ of $\sigma^{\left(\lambda_{\sigma}\right)}(x)$ has a semantic meaning related to the shape of the function curve. The first derivative of $\sigma^{\left(\lambda_{\sigma}\right)}(x)$ at $x=0$ is

$$
\left.\frac{\mathrm{d} \sigma^{\left(\lambda_{\sigma}\right)}(x)}{\mathrm{d} x}\right|_{x=0}=\lambda_{\sigma} \sigma^{\left(\lambda_{\sigma}\right)}(0)\left(1-\sigma^{\left(\lambda_{\sigma}\right)}(0)\right)=\frac{\lambda_{\sigma}}{4} .
$$

That is, the $\lambda_{\sigma}$ parameter determines the slope of $\sigma^{\left(\lambda_{\sigma}\right)}(x)$ at $x=0$.

\section{Convexity.}

- $\sigma^{\left(\lambda_{\sigma}\right)}(x)$ has a single inflection point that is at $x=0$

- If $\lambda_{\sigma}>0$, then $\sigma^{\left(\lambda_{\sigma}\right)}(x)$ changes from concave to convex at $x=0$

- If $\lambda_{\sigma}<0$, then $\sigma^{\left(\lambda_{\sigma}\right)}(x)$ changes from convex to concave at $x=0$

Figure 1 shows some examples of Sigmoid function plots.

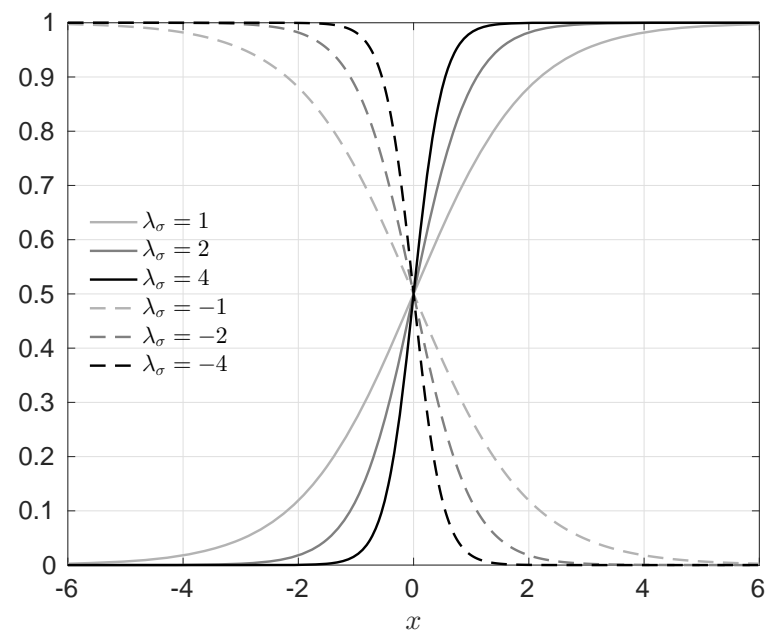

Figure 1: Examples of Sigmoid function plots. 


\subsection{Tocher's Approximation and the Averaging Dombi Conjunction Operator}

Applying the averaging Dombi conjunction in (6) to $\sigma^{\left(\lambda_{\sigma}\right)}(x)$ and $\sigma^{\left(-\lambda_{\sigma}\right)}(x)$ yields the following $d_{\lambda_{\sigma}}(x)$ function:

$$
\begin{gathered}
d_{\lambda_{\sigma}}(x)=\bar{c}\left(\sigma^{\left(\lambda_{\sigma}\right)}(x), \sigma^{\left(-\lambda_{\sigma}\right)}(x)\right)=\frac{1}{1+\frac{1}{2}\left(\frac{1-\sigma^{\left(\lambda_{\sigma}\right)}(x)}{\sigma^{\left(\lambda_{\sigma}\right)}(x)}+\frac{1-\sigma^{\left(-\lambda_{\sigma}\right)}(x)}{\sigma^{\left(-\lambda_{\sigma}\right)}(x)}\right)}= \\
=\frac{1}{1+\frac{1}{2}\left(\mathrm{e}^{-\lambda_{\sigma} x}+\mathrm{e}^{\lambda_{\sigma} x}\right)}=\frac{2 \mathrm{e}^{\lambda_{\sigma} x}}{\left(1+\mathrm{e}^{\lambda_{\sigma} x}\right)^{2}} .
\end{gathered}
$$

Figure 2 shows the averaging Dombi conjunction of two Sigmoid fuzzy membership functions; that is, the intersection of two fuzzy sets that are given by Sigmoid functions: by a decreasing and an increasing Sigmoid function with the same absolute $\lambda_{\sigma}$ parameter values.

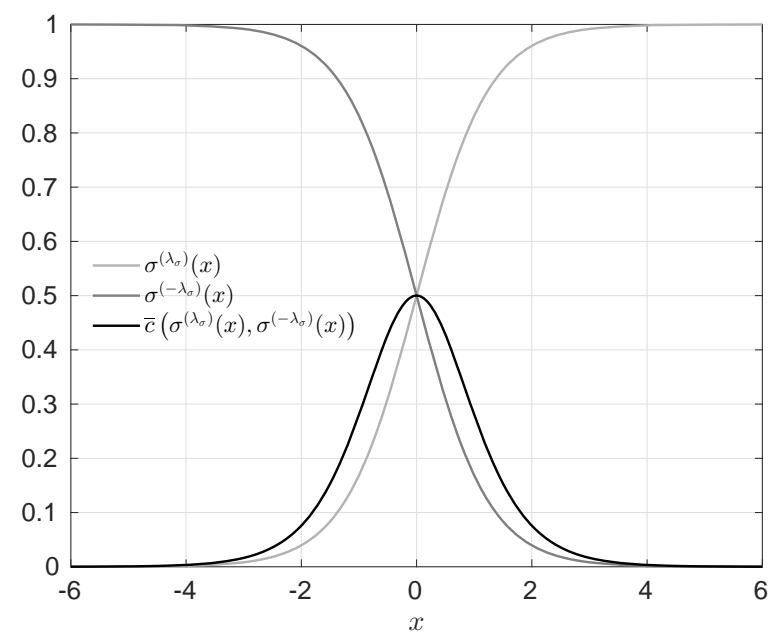

Figure 2: The averaging Dombi conjunction of two Sigmoid fuzzy membership functions.

Function $d_{\lambda_{\sigma}}(x)$, like the density function $\phi(x)$, has a bell-shaped curve, but since

$$
\int_{-\infty}^{+\infty} d_{\lambda_{\sigma}}(x) \mathrm{d} x=\int_{-\infty}^{+\infty} \frac{2 \mathrm{e}^{\lambda_{\sigma} x}}{\left(1+\mathrm{e}^{\lambda_{\sigma} x}\right)^{2}}=\left[-\frac{2}{\lambda_{\sigma}\left(1+\mathrm{e}^{\lambda_{\sigma} x}\right)}\right]_{-\infty}^{+\infty}=\frac{2}{\lambda_{\sigma}},
$$

$d_{\lambda_{\sigma}}(x)$ is not a probability density function. Hence,

$$
\int_{-\infty}^{+\infty} \frac{\lambda_{\sigma}}{2} d_{\lambda_{\sigma}}(x) \mathrm{d} x=\int_{-\infty}^{+\infty} \frac{\lambda_{\sigma} \mathrm{e}^{\lambda_{\sigma} x}}{\left(1+\mathrm{e}^{\lambda_{\sigma} x}\right)^{2}}=1,
$$


and so we define the probability density function $\phi_{\sigma}(x)$ as follows.

Definition 4. The probability density function $\phi_{\sigma}(x)$ is given by

$$
\phi_{\sigma}(x)=\frac{\lambda_{\sigma} \mathrm{e}^{\lambda_{\sigma} x}}{\left(1+\mathrm{e}^{\lambda_{\sigma} x}\right)^{2}},
$$

where $\lambda_{\sigma}=2 \sqrt{2 / \pi}$.

Note that setting $\lambda_{\sigma}$ to $2 \sqrt{2 / \pi}$ ensures that

$$
\left.\phi_{\sigma}(x)\right|_{x=0}=\left.\phi(x)\right|_{x=0} .
$$

The corresponding probability distribution function $\Phi_{\sigma}(x)$ is

$$
\Phi_{\sigma}(x)=\int_{-\infty}^{x} \phi_{\sigma}(t) \mathrm{d} t=\left[-\frac{1}{1+\mathrm{e}^{2 \sqrt{2 / \pi} t}}\right]_{-\infty}^{x}=\frac{1}{1+\mathrm{e}^{-2 \sqrt{2 / \pi} x}} .
$$

This means that the probability distribution function $\Phi_{\sigma}(x)$ is a Sigmoid function that has the parameter $\lambda_{\sigma}=2 \sqrt{2 / \pi}$. It is worth adding here that $\Phi_{\sigma}(x)$ is identical to Tocher's approximation result in (3) from 1963 [29]. However, we derived the function $\Phi_{\sigma}(x)$ by generating the density function $\phi_{\sigma}(x)$ from Sigmoid functions by utilizing the averaging Dombi conjunction operator, and this approach is different from Tocher's.

Approximation accuracy. It can be shown numerically that

$$
\max _{x \in \mathbb{R}}\left|\Phi(x)-\Phi_{\sigma}(x)\right| \approx 0.0177 .
$$

Figure 3 shows the curve of absolute error function $\left|\Phi(x)-\Phi_{\sigma}(x)\right|$.

Properties of the approximation. Here, we summarize the properties of this approximation in the light of expectations that were prescribed in Section 3.1.

- Simplicity and accuracy. $\Phi_{\sigma}(x)$ has a simple formula, but its maximum absolute approximation error has an order of magnitude of -2 .

- Identity to first order at zero. Since $\Phi_{\sigma}(0)=\Phi(0)$ and the parameter $\lambda_{\sigma}$ of $\phi_{\sigma}(x)$ was set such that $\phi_{\sigma}(0)=\phi(0), \Phi_{\sigma}(x)$ and $\Phi(x)$ are identical to first order at $x=0$.

- Symmetry. The probability density function $\phi_{\sigma}(x)$ is an even function and so $\Phi_{\sigma}(-x)=1-\Phi_{\sigma}(x)$ holds for any $x \in \mathbb{R}$.

- Direct connection between the density and distribution functions. There is an interesting relation between the probability density function $\phi_{\sigma}(x)$ 


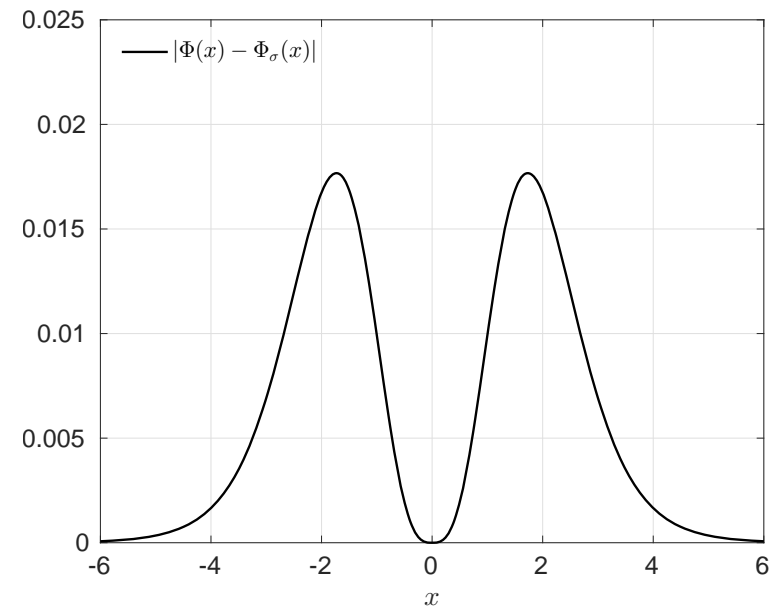

Figure 3: Absolute errors of approximation by $\Phi_{\sigma}(x)$.

and the probability distribution function $\Phi_{\sigma}(x)$ that is worth mentioning here. Namely, utilizing (17) and $\lambda_{\sigma}=2 \sqrt{2 / \pi}$ gives the following equation:

$$
\phi_{\sigma}(x)=2 \sqrt{\frac{2}{\pi}} \Phi_{\sigma}(x)\left(1-\Phi_{\sigma}(x)\right) .
$$

That is, $\phi_{\sigma}(x)$ can be expressed in terms of $\Phi_{\sigma}(x)$ in a closed form.

According to Hillier and Liberman [17], the Sigmoid function that matches $\Phi(x)$ the best, has only one parameter and the form of (10) is

$$
\Phi_{H L}(x)=\frac{1}{1+\mathrm{e}^{-1.702 x}} .
$$

This approximation has a maximum absolute error of 0.0095 . Note that although this approximation yields a higher accuracy than the approximation by the $\Phi_{\sigma}(x)$ function, the first derivative of $\Phi_{H L}(x)$ at $x=0$ is not $1 / \sqrt{2 \pi}$; that is, $\Phi_{H L}(x)$ is not identical with $\Phi(x)$ to first order.

Note that if we used the Dombi conjunction operator in (5) to create a probability density function from two Sigmoid functions, then we would get the following probability distribution function:

$$
\Phi_{\sigma}^{*}(x)=\frac{3}{\pi} \arctan \left(\frac{\sqrt{3}}{3}\left(2 \mathrm{e}^{\sqrt{6 \pi} / 3 x}+1\right)\right)-\frac{1}{2} .
$$

The maximum absolute error of this approximation is 0.0231 and calculation of the approximation formula requires the computation of an exponential function and an arcus tangent function. We can find simpler formulas with better precision values among the known approximations enumerated in Section 2. 


\subsection{An Approximation Connected with the Unary Modifier Operator}

\subsubsection{The Epsilon Function}

Here, we introduce the Epsilon function that we will utilize for constructing approximations to the standard normal probability distribution function.

Definition 5. The Epsilon function $\varepsilon_{d}^{(\lambda)}(x)$ is given by

$$
\varepsilon_{d}^{(\lambda)}(x)=\left(\frac{x+d}{d-x}\right)^{\lambda \frac{d}{2}}
$$

where $\lambda \in \mathbb{R}, \lambda \neq 0, d \in \mathbb{R}, d>0, x \in(-d,+d)$.

The following theorem introduces an important asymptotic property of the Epsilon function.

Theorem 1. For any $x \in(-d,+d)$, if $d \rightarrow \infty$,

$$
\varepsilon_{d}^{(\lambda)}(x) \rightarrow \mathrm{e}^{\lambda x} .
$$

Proof. Let $x$ have a fixed value, $x \in(-d,+d)$.

$$
\begin{gathered}
\lim _{d \rightarrow \infty} \varepsilon_{d}^{(\lambda)}(x)=\lim _{d \rightarrow \infty}\left(\frac{x+d}{d-x}\right)^{\lambda \frac{d}{2}}=\lim _{d \rightarrow \infty}\left(\left(\frac{d-x+2 x}{d-x}\right)^{d}\right)^{\frac{\lambda}{2}}= \\
=\lim _{d \rightarrow \infty}\left(\left(1+\frac{2 x}{d-x}\right)^{d}\right)^{\frac{\lambda}{2}} .
\end{gathered}
$$

Since $x$ is fixed, if $d \rightarrow \infty$, then $\Delta=d-x \rightarrow \infty$ and so the previous equation can be continued as follows:

$$
\begin{gathered}
\lim _{d \rightarrow \infty}\left(\left(1+\frac{2 x}{d-x}\right)^{d}\right)^{\frac{\lambda}{2}}=\lim _{\Delta \rightarrow \infty}\left(\left(1+\frac{2 x}{\Delta}\right)^{\Delta+x}\right)^{\frac{\lambda}{2}}= \\
=\left(\lim _{\Delta \rightarrow \infty}\left(1+\frac{2 x}{\Delta}\right)^{\Delta} \lim _{\Delta \rightarrow \infty}\left(1+\frac{2 x}{\Delta}\right)^{x}\right)^{\frac{\lambda}{2}}=\left(\mathrm{e}^{2 x}\right)^{\frac{\lambda}{2}} \cdot 1^{\frac{\lambda}{2}}=\mathrm{e}^{\lambda x} .
\end{gathered}
$$

Based on Theorem 1, we can state that the asymptotic Epsilon function is just the exponential function. It is worth mentioning here that the Epsilon function is the basis of the so-called Epsilon probability distribution, which can be utilized to approximate the exponential probability distribution [12]. 


\subsubsection{The Kappa Function and Some of its Basic Properties}

Here, we define the Kappa function that we will use to approximate the standard normal probability distribution function.

Definition 6. The Kappa function $\kappa_{d}^{\left(\lambda_{\kappa}\right)}(x)$ is given by

$$
\kappa_{d}^{\left(\lambda_{\kappa}\right)}(x)=\frac{1}{1+\left(\frac{d-x}{d+x}\right)^{\lambda_{\kappa}}},
$$

where $\lambda_{\kappa} \in \mathbb{R}, \lambda_{\kappa}>0, d \in \mathbb{R}, d>0, x \in(-d,+d)$.

Note that we utilize the Kappa function $\kappa_{d}^{\left(\lambda_{\kappa}\right)}(x)$ solely with positive $\lambda_{\kappa}$ parameter values. Here, we state the most important properties of the Kappa function $\kappa_{d}^{\left(\lambda_{\kappa}\right)}(x)$; namely, range, continuity, monotonicity, limits, role of the parameters and convexity.

Range. The range of $\kappa_{d}^{\left(\lambda_{\kappa}\right)}(x)$ is the interval $(0,1]$.

Continuity. $\kappa_{d}^{\left(\lambda_{\kappa}\right)}(x)$ is a continuous function in $(-d,+d)$.

Monotonicity. As $\lambda_{\kappa}>0, \kappa_{d}^{\left(\lambda_{\kappa}\right)}(x)$ is strictly monotonously increasing in the interval $(-d,+d)$.

Limits.

$$
\begin{aligned}
& \lim _{x \rightarrow-d^{+}} \kappa_{d}^{\left(\lambda_{\kappa}\right)}(x)=0 \\
& \lim _{x \rightarrow+d^{-}} \kappa_{d}^{\left(\lambda_{\kappa}\right)}(x)=1
\end{aligned}
$$

Note that as $\lambda_{\kappa}>0, \kappa_{d}^{\left(\lambda_{\kappa}\right)}(x)$ takes the value of 1 at $d$.

Role of the parameters. Both parameters $\lambda_{\kappa}$ and $d$ of $\kappa_{d}^{\left(\lambda_{\kappa}\right)}(x)$ have a semantic meaning related to the shape of the function curve.

- Parameter $d$ specifies the $(-d,+d)$ domain of $\kappa_{d}^{\left(\lambda_{\kappa}\right)}(x)$.

- The first derivative of $\kappa_{d}^{\left(\lambda_{\kappa}\right)}(x)$ at $x=0$ is

$$
\left.\frac{\mathrm{d} \kappa_{d}^{\left(\lambda_{\kappa}\right)}(x)}{\mathrm{d} x}\right|_{x=0}=\left.2 \lambda_{\kappa} d \frac{\kappa_{d}^{\left(\lambda_{\kappa}\right)}(x)\left(1-\kappa_{d}^{\left(\lambda_{\kappa}\right)}(x)\right)}{(d-x)(d+x)}\right|_{x=0}=\frac{\lambda_{\kappa}}{2 d} .
$$

That is, parameter $\lambda_{\kappa}$ determines the gradient of function $\kappa_{d}^{\left(\lambda_{\kappa}\right)}(x)$ at $x=0$. 
Convexity. It can be shown that the Kappa function $\kappa_{d}^{\left(\lambda_{\kappa}\right)}(x)$ has a single inflection point at $x=0$, where it changes its shape from convex to concave.

\subsubsection{Connection with the Sigmoid function}

The Kappa function has the following asymptotic property that allows us to use it for approximating the Sigmoid function and through that the standard normal probability distribution function.

Lemma 1. If $\sigma^{\left(\lambda_{\sigma}\right)}(x)$ is a Sigmoid function with the parameter $\lambda_{\sigma}>0, \kappa_{d}^{\left(\lambda_{\kappa}\right)}(x)$ is a Kappa function with parameters $\lambda_{\kappa}, d>0$ and

$$
\lambda_{\kappa}=\lambda_{\sigma} \frac{d}{2}
$$

then for any $x \in(-d,+d)$, if $d \rightarrow \infty$, then

$$
\kappa_{d}^{\left(\lambda_{\kappa}\right)}(x) \rightarrow \sigma^{\left(\lambda_{\sigma}\right)}(x)
$$

Proof. Let $x$ have a fixed value. If the conditions of the lemma are satisfied, then the Kappa function $\kappa_{d}^{\left(\lambda_{\kappa}\right)}(x)$ may be written as

$$
\kappa_{d}^{\left(\lambda_{\kappa}\right)}(x)=\frac{1}{1+\left(\frac{d-x}{d+x}\right)^{\lambda_{\kappa}}}=\frac{1}{1+\left(\frac{d+x}{d-x}\right)^{-\lambda_{\sigma} \frac{d}{2}}}=\frac{1}{1+\varepsilon_{d}^{\left(-\lambda_{\sigma}\right)}(x)},
$$

and based on Theorem $1, \varepsilon_{d}^{\left(-\lambda_{\sigma}\right)}(x) \rightarrow \mathrm{e}^{-\lambda_{\sigma} x}$, if $d \rightarrow \infty$; that is,

$$
\kappa_{d}^{\left(\lambda_{\kappa}\right)}(x)=\frac{1}{1+\varepsilon_{d}^{\left(-\lambda_{\sigma}\right)}(x)} \underset{d \rightarrow \infty}{\longrightarrow} \frac{1}{1+\mathrm{e}^{-\lambda_{\sigma} x}}=\sigma^{\left(\lambda_{\sigma}\right)}(x) .
$$

Corollary 1. In the interval $(-d,+d)$, the probability distribution function

$$
\Phi_{\sigma}(x)=\frac{1}{1+\mathrm{e}^{-\lambda_{\sigma} x}}
$$

can be approximated by the Kappa function

$$
\kappa_{d}^{\left(\lambda_{\kappa}\right)}(x)=\frac{1}{1+\left(\frac{d-x}{d+x}\right)^{\lambda_{\kappa}}}
$$

where $d \in \mathbb{R}, d>0, \lambda_{\sigma}=2 \sqrt{2 / \pi}, \lambda_{\kappa}=\sqrt{2 / \pi} d$.

Proof. The corollary follows from Lemma 1. 


\subsubsection{The Quasi Logistic Probability Distribution Function}

Now, we will we define the Quasi Logistic probability distribution function by utilizing the Kappa function given in (28).

Definition 7. The Quasi Logistic probability distribution function is given by

$$
\Phi_{\kappa, d}(x)= \begin{cases}0, & \text { if } x \leq-d \\ \kappa_{d}^{\left(\lambda_{\kappa}\right)}(x), & \text { if } x \in(-d,+d) \\ 1, & \text { if } x \geq+d\end{cases}
$$

where $d \in \mathbb{R}, d>0, \lambda_{\kappa}=\sqrt{2 / \pi} d$.

It is worth mentioning here that there is an interesting relation between the Quasi Logistic probability density function $\phi_{\kappa, d}(x)$ and the probability distribution function $\Phi_{\kappa, d}(x)$.

Lemma 2. If $x \in(-d,+d)$, then

$$
\phi_{\kappa, d}(x)=2 d^{2} \sqrt{\frac{2}{\pi}} \frac{\Phi_{\kappa, d}(x)\left(1-\Phi_{\kappa, d}(x)\right)}{(d-x)(d+x)},
$$

where $d \in \mathbb{R}, d>0$.

Proof. Based on the definition of $\Phi_{\kappa, d}(x)$ in $(38)$, if $x \in(-d,+d)$, then

$$
\Phi_{\kappa, d}(x)=\kappa_{d}^{\left(\lambda_{\kappa}\right)}(x) .
$$

Utilizing this equation, $(31)$ and $\lambda_{\kappa}=\sqrt{2 / \pi} d$, we get

$$
\begin{gathered}
\phi_{\kappa, d}(x)=\frac{\mathrm{d} \Phi_{\kappa, d}(x)}{\mathrm{d} x}=\frac{\mathrm{d} \kappa_{d}^{\left(\lambda_{\kappa}\right)}(x)}{\mathrm{d} x}= \\
=2 \lambda_{\kappa} d \frac{\kappa_{d}^{\left(\lambda_{\kappa}\right)}(x)\left(1-\kappa_{d}^{\left(\lambda_{\kappa}\right)}(x)\right)}{(d-x)(d+x)}=2 d^{2} \sqrt{\frac{2}{\pi}} \frac{\Phi_{\kappa, d}(x)\left(1-\Phi_{\kappa, d}(x)\right)}{(d-x)(d+x)} .
\end{gathered}
$$

Utilizing Lemma 2, the Quasi Logistic probability density function $\phi_{\kappa, d}(x)$ for $x \in \mathbb{R}$ is

$$
\phi_{\kappa, d}(x)= \begin{cases}2 d^{2} \sqrt{\frac{2}{\pi} \frac{\kappa_{d}^{\left(\lambda_{\kappa}\right)}(x)\left(1-\kappa_{d}^{\left(\lambda_{\kappa}\right)}(x)\right)}{(d-x)(d+x)},} & \text { if } x \in(-d,+d) \\ 0, & \text { otherwise, }\end{cases}
$$

where $d \in \mathbb{R}, d>0$.

Note that based on the properties of $\kappa_{d}^{\left(\lambda_{\kappa}\right)}(x)$, it can be shown that $\Phi_{\kappa, d}(x)$ is in fact a probability distribution function and $\phi_{\kappa, d}(x)$ is its probability density function. Therefore, the following criteria are met: 
1. $\phi_{\kappa, d}(x) \geq 0$ for any $x \in \mathbb{R}$

2. $\int_{-\infty}^{+\infty} \phi_{\kappa, d}(x) \mathrm{d} x=1$

3. $\int_{-\infty}^{x} \phi_{\kappa, d}(t) \mathrm{d} t=\Phi_{\kappa, d}(x)$.

Corollary 2. The standard normal probability distribution function $\Phi(x)$ can be approximated by the Quasi Logistic probability distribution function $\Phi_{\kappa, d}(x)$.

Proof. The corollary follows from the fact that $\Phi(x)$ can be approximated by the Sigmoid function $\sigma^{\left(\lambda_{\sigma}\right)}(x)$ that has the parameter $\lambda_{\sigma}=2 \sqrt{2 / \pi}$ and from Corollary 1 and from the definition of the Quasi Logistic probability distribution function.

It is worth mentioning that $\phi_{\kappa, d}(x)$ can be derived from the Kappa function also in the following way. Utilizing the fact that

$$
f_{\lambda_{\kappa}, d}(x)= \begin{cases}\frac{\mathrm{d} \kappa_{d}^{\left(\lambda_{\kappa}\right)}(x)}{\mathrm{d} x}, & \text { if } x \in(-d,+d) \\ 0, & \text { otherwise }\end{cases}
$$

is a probability density function,

$$
\frac{\mathrm{d} \kappa_{d}^{\left(\lambda_{\kappa}\right)}(x)}{\mathrm{d} x}=2 \lambda_{\kappa} d \frac{\kappa_{d}^{\left(\lambda_{\kappa}\right)}(x)\left(1-\kappa_{d}^{\left(\lambda_{\kappa}\right)}(x)\right)}{(d-x)(d+x)},
$$

and setting the requirement $f_{\lambda_{\kappa}, d}(0)=\phi(0)$ results in the following equation:

$$
\left.2 \lambda_{\kappa} d \frac{\kappa_{d}^{\left(\lambda_{\kappa}\right)}(x)\left(1-\kappa_{d}^{\left(\lambda_{\kappa}\right)}(x)\right)}{(d-x)(d+x)}\right|_{x=0}=\left.\frac{1}{\sqrt{2 \pi}} \mathrm{e}^{-\frac{x^{2}}{2}}\right|_{x=0} .
$$

Using (31), this equation leads to $\lambda_{\kappa}=\sqrt{2 / \pi} d$; that is, $f_{\lambda_{\kappa}, d}(x)=\phi_{\kappa, d}(x)$, if $\lambda_{\kappa}=\sqrt{2 / \pi} d$.

\subsubsection{Approximation Accuracy}

It can be shown numerically that $\left|\Phi(x)-\Phi_{\kappa, d}(x)\right|$ is approximately minimal, if $d=3.1152$. In this case, the maximum absolute approximation error is $2.15 \cdot 10^{-3}$. Considering the fact that 3.1152 is close to $\pi$, using $d=\pi$ instead of $d=3.1152$ does not worsen significantly the approximation accuracy. If $d=\pi$, then the maximum absolute approximation error is $2.36 \cdot 10^{-3}$. Although the parameter $d$ with value of $d=3.1152$ yields the least maximum absolute approximation error among the Quasi Logistic probability distribution functions, we propose the use of function 
$\Phi_{\kappa, \pi}(x)$ as it has a very simple form and its maximum absolute approximation error is just slightly greater than that of function $\Phi_{\kappa, d}(x)$ with $d=3.1152$.

$$
\Phi_{\kappa, \pi}(x)= \begin{cases}0, & \text { if } x \leq-\pi \\ \frac{1}{1+\left(\frac{\pi-x}{\pi+x}\right) \sqrt{2 \pi}}, & \text { if } x \in(-\pi,+\pi) \\ 1, & \text { if } x \geq+\pi\end{cases}
$$

We call the Quasi Logistic probability distribution function with $d=\pi$; that is, the function $\Phi_{\kappa, \pi}(x)$, the Dombi-Jónás probability distribution function. The absolute errors $\left|\Phi(x)-\Phi_{\kappa, d}(x)\right|$ for $d=3.1152$ and $d=\pi$ are shown in Figure 4.

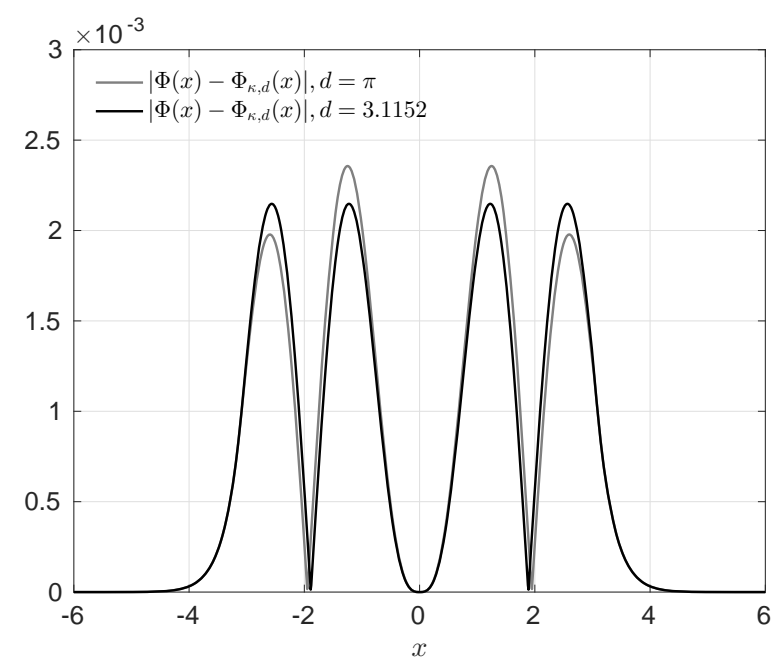

Figure 4: Absolute errors of approximations by using Quasi Logistic probability distribution functions.

\subsubsection{Properties of the Approximation}

Here, we summarize the properties of the $\Phi_{\kappa, \pi}(x)$ approximation.

- Simplicity and accuracy. The maximum absolute error of approximation $\Phi_{\kappa, \pi}(x)$ is $2.36 \cdot 10^{-3}$ and at the same time function $\Phi_{\kappa, \pi}(x)$ has a very simple form. In this accuracy range, there is no other known approximation that has such a simple form. The known approximations that yield higher accuracy have more complex forms, while the ones with similarly complex formulas do not give greater accuracy.

- Identity to first order at zero. Since $\Phi_{\kappa, \pi}(0)=\Phi(0)$ and the probability density function $\phi_{\kappa, \pi}(x)$ was constructed such that $\phi_{\kappa, \pi}(0)=\phi(0), \Phi_{\kappa, \pi}(x)$ and $\Phi(x)$ are identical to first order at $x=0$. 
- Symmetric absolute error function. It can be shown that the probability density function $\phi_{\kappa, \pi}(x)$ is an even function and so $\Phi_{\kappa, \pi}(-x)=1-\Phi_{\kappa, \pi}(x)$ holds for any $x \in \mathbb{R}$.

- Direct connection between the density and distribution functions. Based on Lemma 2, the density function $\phi_{\kappa, \pi}(x)$ can be directly expressed in terms of the distribution function $\Phi_{\kappa, \pi}(x)$ in a closed form.

\subsubsection{Connections with Dombi Operators}

Next, we will show how the Epsilon function $\varepsilon_{d}^{(-\lambda)}(x)$ and the Kappa function $\kappa_{d}^{\left(\lambda_{\kappa}\right)}(x)$ are connected with the Dombi operators.

Lemma 3. The generator function $f_{\alpha}(x)$ of Dombi conjunction and disjunction operators can be derived from the Epsilon function $\varepsilon_{d}^{(-\lambda)}(x)$ by a linear function transformation.

Proof. Let us apply the $x^{\prime}=(x+d) /(2 d)$ linear transformation to the variable $x$, where $x \in(-d, d), d>0$. After this transformation, the domain of $x^{\prime}$ is the interval $(0,1), x=2 d x^{\prime}-d$, and

$$
\begin{gathered}
\varepsilon_{d}^{(-\lambda)}(x)=\left(\frac{x+d}{d-x}\right)^{-\lambda \frac{d}{2}}=\left(\frac{2 d x^{\prime}-d+d}{d-2 d x^{\prime}+d}\right)^{-\lambda \frac{d}{2}}=\left(\frac{x^{\prime}}{1-x^{\prime}}\right)^{-\lambda \frac{d}{2}}= \\
=\left(\frac{1-x^{\prime}}{x^{\prime}}\right)^{\lambda \frac{d}{2}}=f_{\alpha}\left(x^{\prime}\right),
\end{gathered}
$$

where $\alpha=\lambda d / 2$.

Based on this result, the generator function of the Dombi operators may be viewed as a special case of the Epsilon function.

Lemma 4. If $\nu=\nu_{0}=1 / 2$, then the Kappa function $\kappa_{d}^{\left(\lambda_{\kappa}\right)}(x)$ can be derived from the Kappa function $\kappa_{\nu, \nu_{0}}^{(\lambda)}(x)$ in (9) by applying a linear function transformation.

Proof. The lemma can be proven by setting $\lambda=\lambda_{\kappa}$ and applying the $x^{\prime}=2 d x-d$ linear transformation $(d>0)$.

Based on this lemma, we can state that the Kappa function $\kappa_{d}^{\left(\lambda_{\kappa}\right)}(x)$, which we utilized to construct the Quasi Logistic probability distribution function, is a special case of the general fuzzy modifier operators.

\section{Conclusions and Future Work}

Table 1 summarizes the maximum absolute errors of the approximations presented earlier. From this table, we can see that the approximation by function $\Phi_{\kappa, \pi}(x)$ has a one order of magnitude less maximum absolute error than the approximation by 
Table 1: Goodness of the approximations given previously

\begin{tabular}{lc}
$F(x)$ & $\max _{x \in \mathbb{R}}|\Phi(x)-F(x)|$ \\
\hline$\Phi_{\sigma}(x)$ & $1.77 \cdot 10^{-2}$ \\
$\Phi_{\kappa, \pi}(x)$ & $2.36 \cdot 10^{-3}$ \\
\hline
\end{tabular}

the function $\Phi_{\sigma}(x)$. Figure 5 and Figure 6 show the approximating function curves and the absolute errors of the approximations, respectively.

Based on comparisons of these approximations with the ones given in the literature, the following findings should be emphasized.

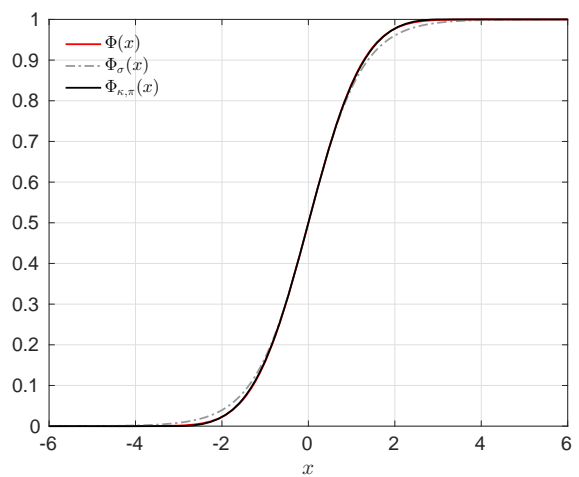

Figure 5: Approximations.

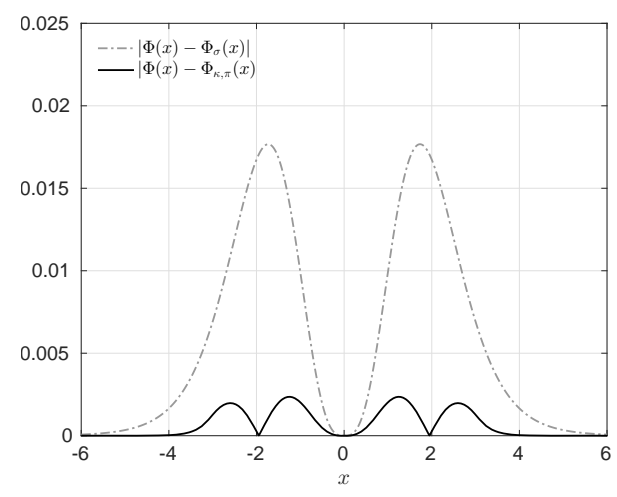

Figure 6: Absolute errors.

Simplicity and accuracy. The first of the approximations listed earlier, which is the same as Tocher's approximation [29], has a maximum absolute approximation error of $1.77 \cdot 10^{-2}$. Although this approximation has a simple form, its accuracy is lower than the accuracy of some known approximations that have similar complex formulas (e.g. [26], [20], [1], [13]). The maximum absolute error of approximation by function $\Phi_{\kappa, \pi}(x)$ is $2.36 \cdot 10^{-3}$. This error is one order of magnitude less than that of the first approximation. At the same time, function $\Phi_{\kappa, \pi}(x)$ has a very simple formula with only one constant parameter which is the constant $\pi$. It should be added here that there are only a few known approximations with a single constant parameter in this accuracy range (e.g. [26], [20], [1], [13]), and all these approximations include exponential terms, while $\Phi_{\kappa, \pi}(x)$ does not contain any. That is, to the best of our knowledge, in this accuracy range, there is no other known approximation that has such a simple formula as $\Phi_{\kappa, \pi}(x)$. The known approximations that yield a higher accuracy have more complex formulas, while the ones with similar complex formulas do not give a higher accuracy. 
Identity to first order at zero. The presented approximations of $\Phi(x)$ are identical with $\Phi(x)$ to first order at $x=0$.

Symmetric absolute error function. It is worth noting that both of the above approximations meet the $F(-x)=1-F(x)$ criterion for any $x \in \mathbb{R}$, and so their absolute error function curves are symmetric with respect to the vertical axis, as can be seen in Figure 6 .

Direct connection between the density and distribution functions. It is the case both for the Sigmoid approximation $\Phi_{\sigma}(x)$ and the Quasi Logistic approximation $\Phi_{\kappa, d}(x)$ that the probability density function can be directly expressed in terms of the probability distribution function in a closed form. That is, the density function can be derived from the distribution function without differentiating it. This property of the of our approximations can be very useful in practice.

Connections with the possibilistic approach. The given approximators are connected with continuous logic. Namely, the approximation $\Phi_{\sigma}(x)$ is derived from Sigmoid fuzzy membership functions by applying the averaging Dombi conjunction operator, while the Quasi Logistic approximation is a linearly transformed form of the Kappa function that is a well-known modifier operator in fuzzy theory.

Applicability. For any $x \in \mathbb{R}$ argument, the standard normal probability distribution function $\Phi(x)$ takes a value in the interval $(0,1)$. In other words, it associates positive probabilities with arguments that are much less than 0 , and gives probabilities less than 1 for those arguments that are much greater than zero. In many practical applications, the probabilities for arguments that are much less or much greater than the expected value of a normally distributed random variable are considered to be zero and one, respectively, although the exact probabilities for these arguments lie in the interval $(0,1)$. The probability distribution function $\Phi_{\kappa, \pi}(x)$ takes a value from the interval $(0,1)$ only if its argument is greater than $-\pi$ and less than $+\pi$. Noting that $\Phi(-\pi)=0.00084, \Phi(\pi)=0.99916$ and

$$
\max _{x \in(-\pi,+\pi)}\left|\Phi(x)-\Phi_{\kappa, \pi}(x)\right| \approx 2.36 \cdot 10^{-3},
$$

the Dombi-Jónás probability distribution may be viewed as an alternative, with bounded domain, to the standard normal probability distribution.

Plans for future work. The Kappa function that we used to construct the probability distribution function $\Phi_{\kappa, \pi}(x)$ is symmetric about the point $(0,0.5)$. In certain economic and technological applications, asymmetric probability distributions with bounded domains are needed for modeling and simulation purposes. As part of our future research work, we would like to study how a generalized, asymmetric version of the Kappa function, which is defined over the bounded domain $(a, b)$, can be utilized for constructing asymmetric probability distribution functions. 


\section{References}

[1] Aludaat, K. M. and Alodat, M. T. A note on approximating the normal distribution function. Applied Mathematical Sciences, 2(9):425-429, 2008.

[2] Bagby, R. J. Calculating normal probabilities. The American Mathematical Monthly, 102:46-49, 1995.

[3] Bowling, S., Khasawneh, M., Kaewkuekool, S., and Cho, B. A logistic approximation to the cumulative normal distribution. Journal of Industrial Engineering and Management, 2(1):114-127, 2009.

[4] Bryc, W. A uniform approximation to the right normal tail integral. Applied Mathematics and Computation, 127(2-3):365-374, 2002.

[5] Choudhury, A. A simple approximation to the area under standard normal curve. Mathematics and Statistics, 2(3):147-149, 2014.

[6] Cuyt, A. A. M., Petersen, V., Verdonk, B., Waadeland, H., and Jones, W. B. Handbook of Continued Fractions for Special Functions. Springer, Berlin/New York, 2008.

[7] Divgi, D. R. Calculation of univariate and bivariate normal probability functions. The Annals of Statistics, 7(4):903-910, 1979.

[8] Dombi, J. A general class of fuzzy operators, the DeMorgan class of fuzzy operators and fuzziness measures induced by fuzzy operators. Fuzzy Sets and Systems, 8(2):149 - 163, 1982.

[9] Dombi, J. Properties of the fuzzy connectives in the light of the general representations theorem. Acta Cybernetica, 7(3):313-321, 1986.

[10] Dombi, J. Towards a general class of operators for fuzzy systems. IEEE Transactions on Fuzzy Systems, 16(2):477-484, 2008.

[11] Dombi, J. On a certain type of unary operators. In 2012 IEEE International Conference on Fuzzy Systems, pages 1-7, June 2012.

[12] Dombi, J., Jónás, T., and Tóth, Zs. E. The epsilon probability distribution and its application in reliability theory. Acta Polytechnica Hungarica, 15(1):197$216,2018$.

[13] Eidous, O. and Al-Salman, S. One-term approximation for normal distribution function. Mathematics and Statistics, 4(1):15-18, 2016.

[14] Hamaker, H.C. Approximating the cumulative normal function and its inverse. Applied Statistics, 27:76-77, 1978.

[15] Hart, R. G. A formula for the approximation of definite integrals of the normal distribution function. Mathematicl Tables and ther Aids to Computation, 11(60):265-265, October 1957. 
[16] Hart, R. G. A close approximation related to the error function. Mathematics of Computation, 20(96):600-602, 1966.

[17] Hillier, F. S. and Lieberman, G. J. Introduction to Operations Research, 7th Ed. McGraw-Hill, New York, USA, 2001.

[18] Lee, Chu-In Charles. On laplace continued fraction for the normal integral. Annals of the Institute of Statistical Mathematics, 44(1):107-120, 1992.

[19] Lin, Jinn-Tyan. Approximating the normal tail probability and its inverse for use on a pocket calculator. Applied Statistics, 38:69-70, 1989.

[20] Lin, Jinn-Tyan. A simpler logistic approximation to the normal tail probability and its inverse. Applied Statistics, 39:255-257, 1990.

[21] Matic, I., Radoicic, R., and Stefanica, D. A sharp Polya-based approximation to the normal cdf. SSRN, 2016. http://dx.doi.org/10.2139/ssrn.2842681.

[22] Moran, P. A. P. Calculation of the normal distribution function. Biometrika, 67:675-676, 1980.

[23] Norton, R. M. Pocket-calculator approximation for areas under the standard normal curve. The American Statistician, 43:24-26, 1989.

[24] Page, E. Approximations to the cumulative normal function and its inverse for use on a pocket calculator. Applied Statistics, 26:75-76, 1977.

[25] Patel, J. K. and Read, C. B. Handbook of the normal distribution. Marcel Dekker Inc, 1996.

[26] Pólya, G. Remarks on computing the probability integral in one and two dimensions. In Proceedings of the 1st Berkeley Symposium on Mathematical Statistics and Probability, pages 63-78, Berkeley, Calif., 1949. University of California Press.

[27] Shore, Ha. Accurate RMM-based approximations for the CDF of the normal distribution. Communications in Statistics - Theory and Methods, 34(3):507513, 2005.

[28] Soranzo, A. and Epure, E. Very simply explicitly invertible approximations of normal cumulative and normal quantile function. Applied Mathematical Sciences, 8(87):4323-4341, 2014.

[29] Tocher, K. D. The Art of Simulation. English University Press, London, 1963.

[30] Vazquez-Leal, H., Castaneda-Sheissa, R., Filobello-Nino, U., Sarmiento-Reyes, A., and Orea, J. Sanchez. High accurate simple approximation of normal distribution integral. Mathematical Problems in Engineering, 2012(ID:124029), 2012. 
[31] Waissi, Gary R. and Rossin, Donald F. A sigmoid approximation of the standard normal integral. Applied Mathematics and Computation, 77:91-95, 1996.

[32] Yerukala, R. and Boiroju, N. K. Approximating to the cumulative normal function and its inverse. International Journal of Scientific 85 Engineering Research, 6(4):515-518, 2015.

[33] Yerukala, R., Boiroju, N. K., and Reddy, M. K. An approximation to the cdf of standard normal distribution. International Journal of Mathematical Archive, 2(7):1077-1079, 2011.

[34] Zelen, M. and Severo, N. C. Probability functions. In Abramowitz, M. and Stegun, I. A., editors, Handbook of Mathematical Functions with Formulas, Graphs, and Mathematical Tables, Applied Mathematics Series, pages 925995. Washington:National Bureau of Standards, 1964.

Received 25th February 2018 